\title{
Newsfronts
}

\section{A Gut Instinct to Fight Infection}

Your most powerful defense against the perils of undercooked chicken may already be hidden in your gut. A recent study has found that a naturally produced intestinal peptide can have a substantial impact on the body's ability to fight off Salmonella infection, as demonstrated in a transgenic mouse model.

Researchers at the Medical College of Wisconsin (Milwaukee) and Lerner Research Institute (Cleveland, $\mathrm{OH}$ ) have developed a transgenic mouse line that overexpresses HD-5, a human peptide normally produced in specialized niches within the small intestinal wall by cells known as Paneth cells. These cells store HD-5 as an inactive propeptide, which undergoes cleavage before secretion into the intestinal lumen. Thus activated, HD-5 is thought to play an important role in the body's innate defenses against bacterial infection.

Confirming this theory were studies showing that the HD-5 transgenics showed considerably elevated resistance to the ravages of Salmonella typhimurium infection (Nature, 3 April). Transgenic mice treated with $10^{8}$ colony-forming units (c.f.u.) of virulent bacteria retained considerably lower densities of intestinal S. typhimurium than wild-type animals. After challenge with greater numbers of bacteria $\left(1.5 \times 10^{9}\right.$ c.f.u. $)$, wild-type mice showed significant external signs of illness, and all died within $24 \mathrm{~h}$ of oral inoculation. By comparison, the transgenic mice showed milder symptoms, and all mice recovered within $12 \mathrm{~h}$ after the initial inoculation. Biochemical studies showed that the transgenic mice were properly expressing and processing HD-5; furthermore, the peptide's ability to combat $S$. typhimurium infection was eliminated when the bacteria were introduced intraperitoneally, confirming that the small intestine is $\mathrm{HD}-5$ 's primary site of action.

These results complement a growing body of data supporting an important role for mammalian antimicrobial peptides in staving off bacterial infection, and suggest a potentially valuable therapeutic target for the augmentation of innate host immunity. - Michael Eisenstein

\section{Bird Brain Suggests Origin of Brain Sex Differences}

By studying a rare hermaphroditic bird, researchers have shown that the sexual genotype of brain cells contributes directly to sex differences in the brains of birds, beyond the well-established action of sex hormones.

Although it is accepted that genes on the sex chromosomes determine whether an animal will develop male or female reproductive organs, the origin of sex differences in brain function remains unclear. There have been suggestions that somatic sexual differentiation results exclusively from the effects of circulating sex hormones, rather than from the direct effects of sex chromosome genes. However, recent studies, in which researchers manipulated sex hormone levels

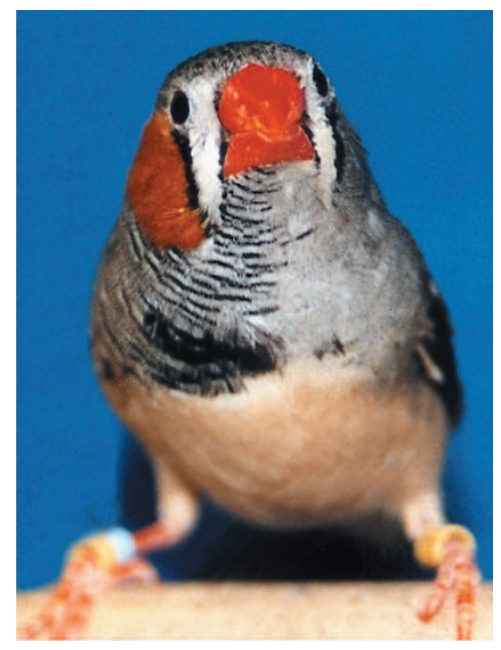

Photo @National Academy of Sciences in developing birds, have suggested that genes may be active in sex determination as well. The discovery of a bilateral gynandromorphic zebra finch (Taeniopygia guttata), which exhibited male characteristics on the right side of its body and female characteristics on the left, provided researchers with the perfect opportunity to test this hypothesis.

This odd bird, the result of an unknown developmental error, was hatched in a colony at Rockefeller University (New York, NY) and given to a group led by Arthur P. Arnold at the University of California, Los Angeles for analysis. Although the bird's behavior was male in that it copulated with females and sang courtship songs, Arnold's group found that the bird's sexual genotype and phenotype were laterally divided throughout its body (Proc. Natl. Acad. Sci. USA, published online, doi: 10.1073/pnas.0636925100).

In addition to having characteristically male brightly colored plumage on the right and dull typically female feathers on the left, the finch's song circuit-the brain area responsible for the males' singing of courtship songs-was $82 \%$ larger on the "male" side than on the "female" side. The bird's vocal organ was intermediate in size relative to male and female controls. Genomic PCR and mRNA analysis revealed that cells on the bird's right side were genetically male, while those on the left side were genetically female; furthermore, female sex chromosome-linked genes were only expressed on the left side.

Because both halves of the finch's brain were exposed to the same hormonal environment, "We now suspect, based on this and other work, that the sex chromosomes present within brain cells also help cause sex differences in brain development," Arnold tells Lab Animal. He goes on to caution, "One can't immediately generalize these results to other species .... However, there is evidence from studies on mammals that supports the same idea." -Tanja Schub

\section{Why Can't Johnny Clone?}

A recently published study has shed some new light on the mystery of why clones derived from certain cell types fare significantly better than others.

In recent years there has been a rapid and highly controversial emergence of mammalian cloning technology. The technique of nuclear transfer-first described half a century ago in experiments with frogs - remains the linchpin of these modern cloning methodologies. This system involves transplanting a nucleus isolated from a "donor" cell into a denucleated, fertilized oocyte, which then undergoes normal division and development with the donor's genetic content. However, the efficiency of this technique depends heavily on the developmental stage of the donor nucleus, and only $1-3 \%$ of blastocysts derived from mature somatic cells will grow to term.

Rudolf Jaenisch's group at MIT (Cambridge, MA) decided to investigate the 